\title{
An Introduction to Probabilistic modeling
}

\section{Oliver Stegle and Karsten Borgwardt}

\author{
Machine Learning and \\ Computational Biology Research Group, \\ Max Planck Institute for Biological Cybernetics and \\ Max Planck Institute for Developmental Biology, Tübingen
}




\section{Why probabilistic modeling?}

- Inferences from data are intrinsically uncertain. 


\section{Why probabilistic modeling?}

- Inferences from data are intrinsically uncertain.

- Probability theory: model uncertainty instead of ignoring it!

- Applications: Machine learning, Data Mining, Pattern Recognition, etc. 


\section{Why probabilistic modeling?}

- Inferences from data are intrinsically uncertain.

- Probability theory: model uncertainty instead of ignoring it!

- Applications: Machine learning, Data Mining, Pattern Recognition, etc.

- Goal of this part of the course

- Overview on probabilistic modeling

- Key concepts

- Focus on Applications in Bioinformatics 


\section{Further reading, useful material}

- Christopher M. Bishop: Pattern Recognition and Machine learning.

- Good background, covers most of the course material and much more!

- Substantial parts of this tutorial borrow figures and ideas from this book.

- David J.C. MacKay: Information Theory, Learning and Inference

- Very worth while reading, not quite the same quality of overlap with the lecture synopsis.

- Freely available online. 


\section{Lecture overview}

1. An Introduction to probabilistic modeling

2. Applications: linear models, hypothesis testing

3. An introduction to Gaussian processes

4. Applications: time series, model comparison

5. Applications: continued 


\section{Outline}




\section{Outline}

\section{Motivation}

\section{Prerequisites}

Probability Theory

Parameter Inference for the Gaussian

Summary 


\section{Key concepts}

Data

- Let $\mathcal{D}$ denote a dataset, consisting of $N$ datapoints

$$
\mathcal{D}=\{\underbrace{\mathbf{x}_{n}}_{\text {Inputs }}, \underbrace{y_{n}}_{\text {Outputs }}\}_{n=1}^{N} \text {. }
$$




\section{Key concepts}

\section{Data}

- Let $\mathcal{D}$ denote a dataset, consisting of $N$ datapoints $\mathcal{D}=\{\underbrace{\mathbf{x}_{n}}, \underbrace{y_{n}}\}_{n=1}^{N}$.

Inputs Outputs

- Typical (this course)

- $\mathbf{x}=\left\{x_{1}, \ldots, x_{D}\right\}$ multivariate, spanning $D$ features for each observation (nodes in a graph, etc.).

- $y$ univariate (fitness, expression level etc.).

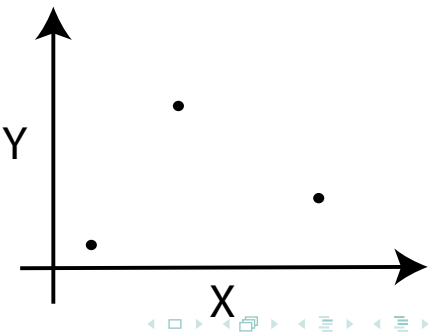




\section{Key concepts}

\section{Data}

- Let $\mathcal{D}$ denote a dataset, consisting of $N$ datapoints $\mathcal{D}=\{\underbrace{\mathbf{x}_{n}}_{\text {Inputs }}, \underbrace{y_{n}}_{\text {Outputs }}\}_{n=1}^{N}$.

- Typical (this course)

- $\mathbf{x}=\left\{x_{1}, \ldots, x_{D}\right\}$ multivariate, spanning $D$ features for each observation (nodes in a graph, etc.).

- $y$ univariate (fitness, expression level etc.).

- Notation:

- Scalars are printed as $y$.

- Vectors are printed in bold: $\mathbf{x}$.

- Matrices are printed in capital bold: $\boldsymbol{\Sigma}$.

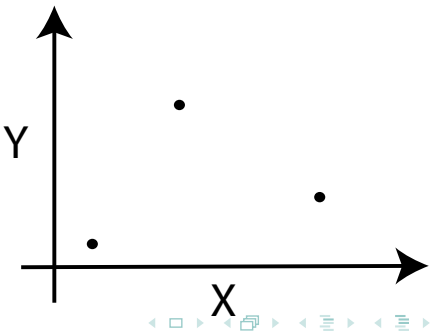




\section{Key concepts

- Observed dataset $\mathcal{D}=\{\underbrace{\mathbf{x}_{n}}, \underbrace{y_{n}}\}_{n=1}^{N}$.

Inputs Outputs

- Given $\mathcal{D}$, what can we say about $y^{\star}$ at an unseen test input $\mathrm{x}^{\star}$ ?

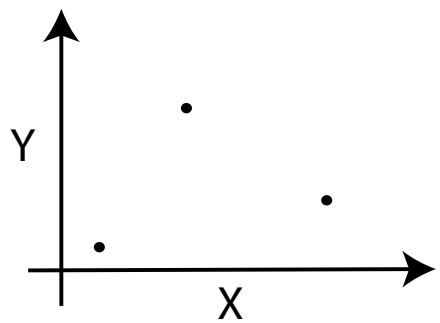




\section{Key concepts

- Observed dataset $\mathcal{D}=\{\underbrace{\mathbf{x}_{n}}, \underbrace{y_{n}}\}_{n=1}^{N}$.

Inputs Outputs

- Given $\mathcal{D}$, what can we say about $y^{\star}$ at an unseen test input $\mathbf{x}^{\star}$ ?

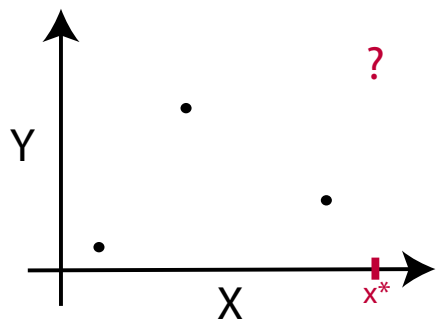




\section{Key concepts \\ Model}

- Observed dataset $\mathcal{D}=\{\underbrace{\mathbf{x}_{n}}_{\text {Inputs }}, \underbrace{y_{n}}_{\text {Outputs }}\}_{n=1}^{N}$.

- Given $\mathcal{D}$, what can we say about $y^{\star}$ at an unseen test input $\mathbf{x}^{\star}$ ?

- To make predictions we need to make assumptions.

- A model $\mathcal{H}$ encodes these assumptions and often depends on some parameters $\boldsymbol{\theta}$.

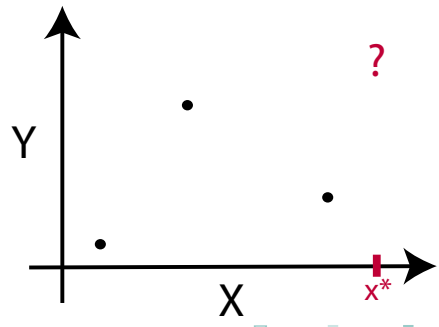




\section{Key concepts}

\section{Model}

- Observed dataset $\mathcal{D}=\{\underbrace{\mathbf{x}_{n}}, \underbrace{y_{n}}\}_{n=1}^{N}$.

Inputs Outputs

- Given $\mathcal{D}$, what can we say about $y^{\star}$ at an unseen test input $\mathbf{x}^{\star}$ ?

- To make predictions we need to make assumptions.

- A model $\mathcal{H}$ encodes these assumptions and often depends on some parameters $\boldsymbol{\theta}$.

- Curve fitting: the model relates $\mathrm{x}$ to $\mathrm{y}$,

$$
\begin{aligned}
y & =f(x \mid \boldsymbol{\theta}) \\
& =\underbrace{\theta_{0}+\theta_{1} \cdot x}_{\text {example, a linear model }}
\end{aligned}
$$

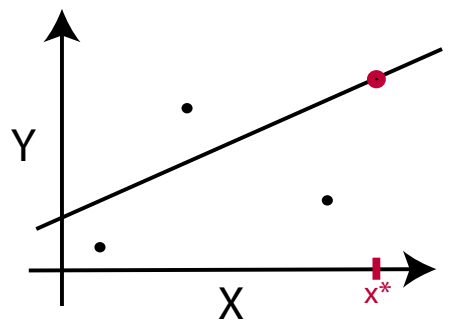




\section{Key concepts}

\section{Uncertainty}

- Virtually in all steps there is uncertainty

- Measurement uncertainty $(\mathcal{D})$

- Parameter uncertainty $(\boldsymbol{\theta})$

- Uncertainty regarding the correct model $(\mathcal{H})$

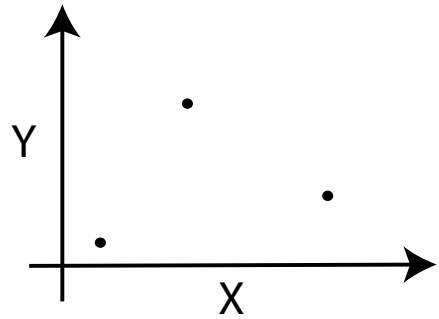




\section{Key concepts}

\section{Uncertainty}

- Virtually in all steps there is uncertainty

- Measurement uncertainty $(\mathcal{D})$

- Parameter uncertainty $(\boldsymbol{\theta})$

- Uncertainty regarding the correct model $(\mathcal{H})$

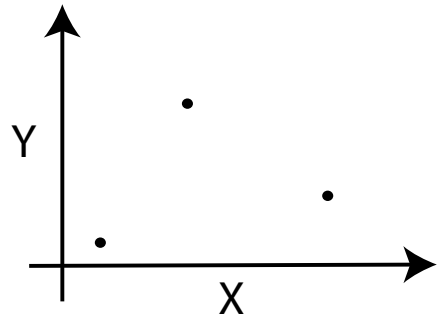




\section{Key concepts \\ Uncertainty}

- Virtually in all steps there is uncertainty

- Measurement uncertainty $(\mathcal{D})$

- Parameter uncertainty $(\boldsymbol{\theta})$

- Uncertainty regarding the correct model $(\mathcal{H})$

Measurement uncertainty

- Uncertainty can occur in both inputs and outputs.

- How to represent uncertainty?

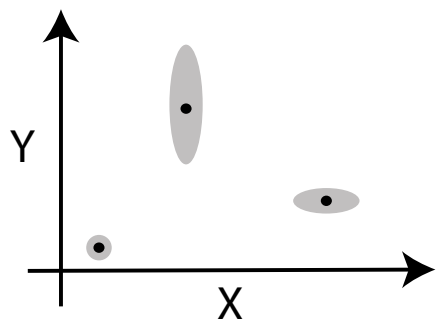




\section{Outline}

Motivation
Prerequisites

Probability Theory

Parameter Inference for the Gaussian

Summary 


\section{Probabilities}

- Let $X$ be a random variable, defined over a set $\mathcal{X}$ or measurable space.

- $P(X=x)$ denotes the probability that $X$ takes value $x$, short $p(x)$.

- Probabilities are positive, $P(X=x) \geq 0$

- Probabilities sum to one

$$
\int_{x \in \mathcal{X}} p(x) d x=1 \quad \sum_{x \in \mathcal{X}} p(x)=1
$$

- Special case: no uncertainty $p(x)=\delta(x-\hat{x})$. 


\section{Probability Theory}

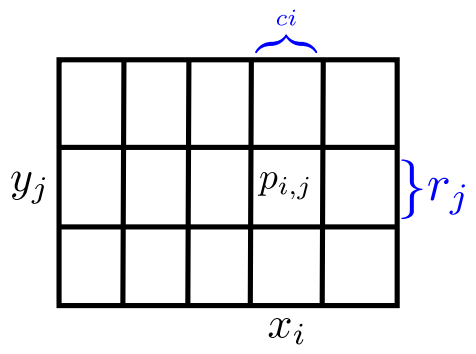

Joint Probability

$$
P\left(X=x_{i}, Y=y_{j}\right)=\frac{n_{i, j}}{N}
$$

\section{Marginal Probability}

$$
P\left(X=x_{i}\right)=\frac{c_{i}}{N}
$$

Conditional Probability

$$
P\left(Y=y_{j} \mid X=x_{i}\right)=\frac{n_{i, j}}{c_{i}}
$$

(C.M. Bishop, Pattern Recognition and Machine Learning) 


\section{Probability Theory}

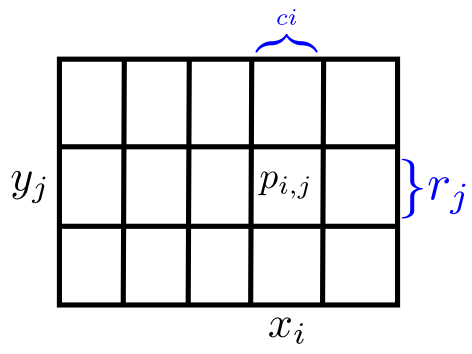

Product Rule

$$
\begin{gathered}
P\left(X=x_{i}, Y=y_{j}\right)=\frac{n_{i, j}}{N}=\frac{n_{i, j}}{c_{i}} \cdot \frac{c_{i}}{N} \\
\quad=P\left(Y=y_{j} \mid X=x_{i}\right) P\left(X=x_{i}\right)
\end{gathered}
$$

\section{Marginal Probability}

$$
P\left(X=x_{i}\right)=\frac{c_{i}}{N}
$$

Conditional Probability

$$
P\left(Y=y_{j} \mid X=x_{i}\right)=\frac{n_{i, j}}{c_{i}}
$$

(C.M. Bishop, Pattern Recognition and Machine Learning) 


\section{Probability Theory}

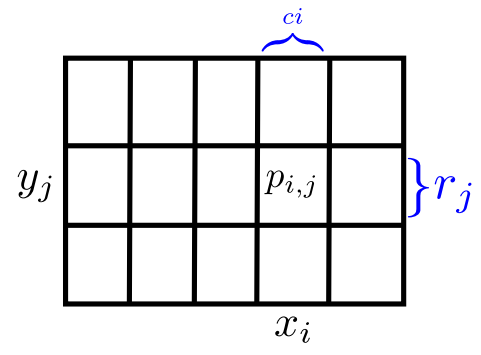

Product Rule

\section{Sum Rule}

$$
\begin{aligned}
P\left(X=x_{i}\right) & =\frac{c_{i}}{N}=\frac{1}{N} \sum_{j=1}^{L} n_{i, j} \\
& =\sum_{j} P\left(X=x_{i}, Y=y_{j}\right)
\end{aligned}
$$

$$
\begin{gathered}
P\left(X=x_{i}, Y=y_{j}\right)=\frac{n_{i, j}}{N}=\frac{n_{i, j}}{c_{i}} \cdot \frac{c_{i}}{N} \\
\quad=P\left(Y=y_{j} \mid X=x_{i}\right) P\left(X=x_{i}\right)
\end{gathered}
$$

(C.M. Bishop, Pattern Recognition and Machine Learning) 


\title{
The Rules of Probability
}

\section{Sum \& Product Rule}

\author{
Sum Rule $\quad p(x)=\sum_{y} p(x, y)$ \\ Product Rule $\quad p(x, y)=p(y \mid x) p(x)$
}




\section{The Rules of Probability}

\section{Bayes Theorem}

- Using the product rule we obtain

$$
\begin{aligned}
p(y \mid x) & =\frac{p(x \mid y) p(y)}{p(x)} \\
p(x) & =\sum_{y} p(x \mid y) p(y)
\end{aligned}
$$




\section{Bayesian probability calculus}

- Bayes rule is the basis for inference and learning.

- Assume we have a model with parameters $\boldsymbol{\theta}$, e.g.

$$
y=\theta_{0}+\theta_{1} \cdot x
$$

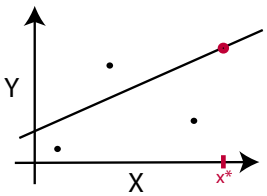

- Goal: learn parameters $\boldsymbol{\theta}$ given Data $\mathcal{D}$.

$$
p(\boldsymbol{\theta} \mid \mathcal{D})=\frac{p(\mathcal{D} \mid \boldsymbol{\theta}) p(\boldsymbol{\theta})}{p(\mathcal{D})}
$$




\section{Bayesian probability calculus}

- Bayes rule is the basis for inference and learning.

- Assume we have a model with parameters $\boldsymbol{\theta}$, e.g.

$$
y=\theta_{0}+\theta_{1} \cdot x
$$

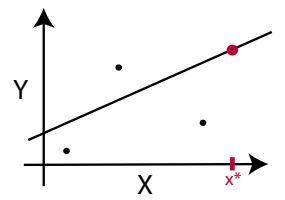

- Goal: learn parameters $\boldsymbol{\theta}$ given Data $\mathcal{D}$.

$$
p(\boldsymbol{\theta} \mid \mathcal{D})=\frac{p(\mathcal{D} \mid \boldsymbol{\theta}) p(\boldsymbol{\theta})}{p(\mathcal{D})}
$$

posterior $\propto$ likelihood $\cdot$ prior

- Posterior

- Likelihood

- Prior 


\section{Information and Entropy}

- Information is the reduction of uncertainty.

- Entropy $H(X)$ is the quantitative description of uncertainty

- $H(X)=0$ : certainty about $\mathrm{X}$.

- $H(X)$ maximal if all possibilities are equal probable.

- Uncertainty and information are additive. 


\section{Information and Entropy}

- Information is the reduction of uncertainty.

- Entropy $H(X)$ is the quantitative description of uncertainty

- $H(X)=0$ : certainty about $\mathrm{X}$.

- $H(X)$ maximal if all possibilities are equal probable.

- Uncertainty and information are additive.

- These conditions are fulfilled by the entropy function:

$$
H(X)=-\sum_{x \in \mathcal{X}} P(X=x) \log P(X=x)
$$




\section{Definitions related to entropy and information}

- Entropy is the average surprise

$$
H(X)=\sum_{x \in \mathcal{X}} P(X=x) \underbrace{(-\log P(X=x))}_{\text {surprise }}
$$




\section{Definitions related to entropy and information}

- Entropy is the average surprise

$$
H(X)=\sum_{x \in \mathcal{X}} P(X=x) \underbrace{(-\log P(X=x))}_{\text {surprise }}
$$

- Conditional entropy

$$
H(X \mid Y)=-\sum_{x \in \mathcal{X}, y \in \mathcal{Y}} P(X=x, Y=y) \log P(X=x \mid Y=y)
$$




\section{Definitions related to entropy and information}

- Entropy is the average surprise

$$
H(X)=\sum_{x \in \mathcal{X}} P(X=x) \underbrace{(-\log P(X=x))}_{\text {surprise }}
$$

- Conditional entropy

$$
H(X \mid Y)=-\sum_{x \in \mathcal{X}, y \in \mathcal{Y}} P(X=x, Y=y) \log P(X=x \mid Y=y)
$$

- Mutual information

$$
\begin{gathered}
I(X: Y)=H(X)-H(X \mid Y)=H(Y)-H(Y \mid X) \\
H(X)+H(Y)-H(X, Y)
\end{gathered}
$$




\section{Definitions related to entropy and information}

- Entropy is the average surprise

$$
H(X)=\sum_{x \in \mathcal{X}} P(X=x) \underbrace{(-\log P(X=x))}_{\text {surprise }}
$$

- Conditional entropy

$$
H(X \mid Y)=-\sum_{x \in \mathcal{X}, y \in \mathcal{Y}} P(X=x, Y=y) \log P(X=x \mid Y=y)
$$

- Mutual information

$$
\begin{gathered}
I(X: Y)=H(X)-H(X \mid Y)=H(Y)-H(Y \mid X) \\
H(X)+H(Y)-H(X, Y)
\end{gathered}
$$

- Independence of $X$ and $Y, p(x, y)=p(x) p(y)$. 


\section{Entropy in action}

\section{The optimal weighting problem}

- Given 12 balls, all equal except for one that is lighter or heavier.

- What is the ideal weighting strategy and how many weightings are needed to identify the odd ball?

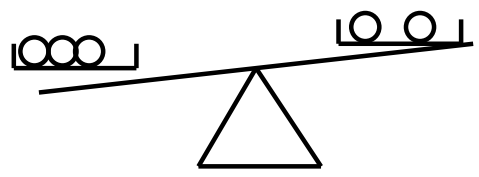




\section{Probability distributions}

- Gaussian

$p\left(x \mid \mu, \sigma^{2}\right)=\mathcal{N}(x \mid \mu, \sigma)=\frac{1}{\sqrt{2 \pi \sigma^{2}}} e^{-\frac{1}{2 \sigma^{2}}(x-\mu)^{2}}$

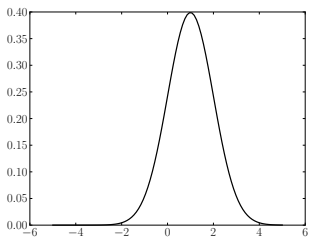




\section{Probability distributions}

- Gaussian

$p\left(x \mid \mu, \sigma^{2}\right)=\mathcal{N}(x \mid \mu, \sigma)=\frac{1}{\sqrt{2 \pi \sigma^{2}}} e^{-\frac{1}{2 \sigma^{2}}(x-\mu)^{2}}$

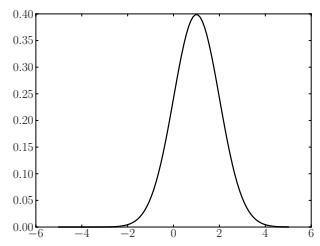

- Multivariate Gaussian

$$
\begin{aligned}
& p(x \mid \boldsymbol{\mu}, \boldsymbol{\Sigma})=\mathcal{N}(\mathbf{x} \mid \boldsymbol{\mu}, \boldsymbol{\Sigma}) \\
& \quad=\frac{1}{\sqrt{|2 \pi \boldsymbol{\Sigma}|}} \exp \left[-\frac{1}{2}(\mathbf{x}-\boldsymbol{\mu})^{\mathrm{T}} \boldsymbol{\Sigma}^{-1}(\mathbf{x}-\boldsymbol{\mu})\right]
\end{aligned}
$$

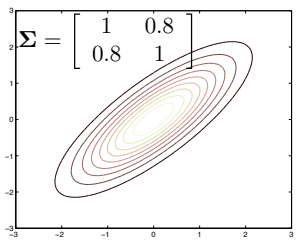




\section{Probability distributions}

\section{continued...}

- Bernoulli

$$
p(x \mid \theta)=\theta^{x}(1-\theta)^{1-x}
$$




\section{Probability distributions}

\section{continued...}

- Bernoulli

$$
p(x \mid \theta)=\theta^{x}(1-\theta)^{1-x}
$$

- Gamma

$$
p(x \mid a, b)=\frac{b^{a}}{\Gamma(a)} x^{a-1} e^{-b x}
$$

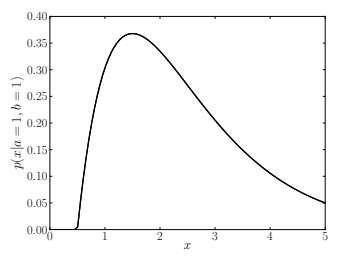




\section{Probability distributions}

\section{The Gaussian revisited}

- Gaussian PDF

$$
\mathcal{N}\left(x \mid \mu, \sigma^{2}\right)=\frac{1}{\sqrt{2 \pi \sigma^{2}}} e^{-\frac{1}{2 \sigma^{2}}(x-\mu)^{2}}
$$

- Positive: $\mathcal{N}\left(x \mid \mu, \sigma^{2}\right)>0$

- Normalized: $\int_{-\infty}^{+\infty} \mathcal{N}(x \mid \mu, \sigma) \mathrm{d} x=1$ (check)

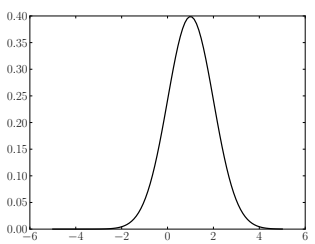




\section{Probability distributions}

\section{The Gaussian revisited}

- Gaussian PDF

$$
\mathcal{N}\left(x \mid \mu, \sigma^{2}\right)=\frac{1}{\sqrt{2 \pi \sigma^{2}}} e^{-\frac{1}{2 \sigma^{2}}(x-\mu)^{2}}
$$

- Positive: $\mathcal{N}\left(x \mid \mu, \sigma^{2}\right)>0$

- Normalized: $\int_{-\infty}^{+\infty} \mathcal{N}(x \mid \mu, \sigma) \mathrm{d} x=1$ (check)

- Expectation:

$$
<x>=\int_{-\infty}^{+\infty} \mathcal{N}\left(x \mid \mu, \sigma^{2}\right) x \mathrm{~d} x=\mu
$$

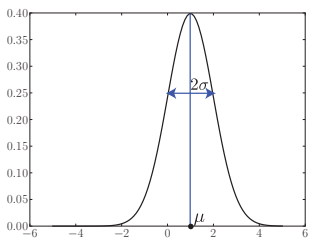

- Variance: $\left.\operatorname{Var}[x]=<x^{2}>-<x\right\rangle^{2}$

$=\mu^{2}+\sigma^{2}-\mu^{2}=\sigma^{2}$ 


\section{Outline}

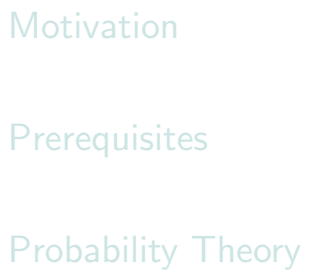




\section{Inference for the Gaussian}

\section{Ingredients}

- Data

$$
\mathcal{D}=\left\{x_{1}, \ldots, x_{N}\right\}
$$

- Model HGauss - Gaussian PDF

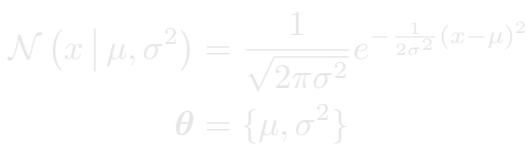

- Likelihood 


\section{Inference for the Gaussian}

\section{Ingredients}

- Data

$$
\mathcal{D}=\left\{x_{1}, \ldots, x_{N}\right\}
$$

- Model $\mathcal{H}_{\text {Gauss }}$ - Gaussian PDF

$$
\begin{aligned}
\mathcal{N}\left(x \mid \mu, \sigma^{2}\right) & =\frac{1}{\sqrt{2 \pi \sigma^{2}}} e^{-\frac{1}{2 \sigma^{2}}(x-\mu)^{2}} \\
\boldsymbol{\theta} & =\left\{\mu, \sigma^{2}\right\}
\end{aligned}
$$

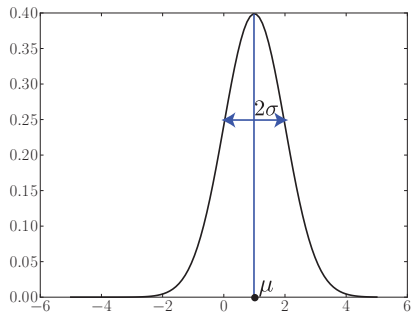




\section{Inference for the Gaussian}

\section{Ingredients}

- Data

$$
\mathcal{D}=\left\{x_{1}, \ldots, x_{N}\right\}
$$

- Model $\mathcal{H}_{\text {Gauss }}$ - Gaussian PDF

$$
\begin{aligned}
\mathcal{N}\left(x \mid \mu, \sigma^{2}\right) & =\frac{1}{\sqrt{2 \pi \sigma^{2}}} e^{-\frac{1}{2 \sigma^{2}}(x-\mu)^{2}} \\
\boldsymbol{\theta} & =\left\{\mu, \sigma^{2}\right\}
\end{aligned}
$$

- Likelihood

$$
p(\mathcal{D} \mid \boldsymbol{\theta})=\prod_{n=1}^{N} \mathcal{N}\left(x_{n} \mid \mu, \sigma^{2}\right)
$$

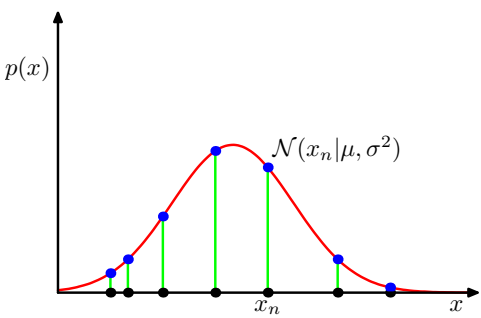

(C.M. Bishop, Pattern Recognition and Machine 


\section{Inference for the Gaussian}

\section{Maximum likelihood}

- Likelihood

$$
p(\mathcal{D} \mid \boldsymbol{\theta})=\prod_{n=1}^{N} \mathcal{N}\left(x_{n} \mid \mu, \sigma^{2}\right)
$$

- Maximum likelihood

$$
\hat{\boldsymbol{\theta}}=\underset{\boldsymbol{\theta}}{\operatorname{argmax}} p(\mathcal{D} \mid \boldsymbol{\theta})
$$

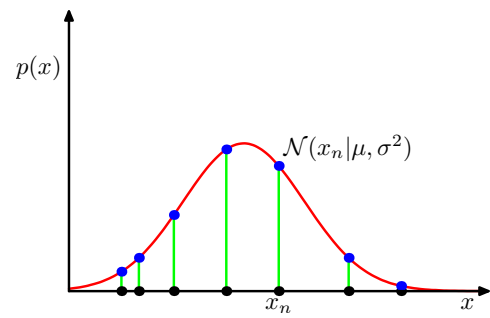

(C.M. Bishop, Pattern Recognition and Machine Learning) 


\section{Inference for the Gaussian}

\section{Maximum likelihood}

$$
\hat{\boldsymbol{\theta}}=\underset{\boldsymbol{\theta}}{\operatorname{argmax}} p(\mathcal{D} \mid \boldsymbol{\theta}) \quad=\underset{\boldsymbol{\theta}}{\operatorname{argmax}} \prod_{n=1}^{N} \frac{1}{\sqrt{2 \pi \sigma^{2}}} e^{-\frac{1}{2 \sigma^{2}}\left(x_{n}-\mu\right)^{2}}
$$




\section{Inference for the Gaussian}

\section{Maximum likelihood}

$$
\hat{\boldsymbol{\theta}}=\underset{\boldsymbol{\theta}}{\operatorname{argmax}} \ln p(\mathcal{D} \mid \boldsymbol{\theta}) \quad=\underset{\boldsymbol{\theta}}{\operatorname{argmax}} \ln \prod_{n=1}^{N} \frac{1}{\sqrt{2 \pi \sigma^{2}}} e^{-\frac{1}{2 \sigma^{2}}\left(x_{n}-\mu\right)^{2}}
$$




\section{Inference for the Gaussian}

\section{Maximum likelihood}

$$
\hat{\boldsymbol{\theta}}=\underset{\boldsymbol{\theta}}{\operatorname{argmax}} \ln p(\mathcal{D} \mid \boldsymbol{\theta})=\underset{\boldsymbol{\theta}}{\operatorname{argmax}}\left[-\frac{N}{2} \ln (2 \pi)-\frac{N}{2} \ln \sigma^{2}-\frac{1}{2 \sigma^{2}} \sum_{n=1}^{N}\left(x_{n}-\mu\right)^{2}\right]
$$




\section{Inference for the Gaussian}

\section{Maximum likelihood}

$\hat{\boldsymbol{\theta}}=\underset{\boldsymbol{\theta}}{\operatorname{argmax}} \ln p(\mathcal{D} \mid \boldsymbol{\theta})=\underset{\boldsymbol{\theta}}{\operatorname{argmax}}\left[-\frac{N}{2} \ln (2 \pi)-\frac{N}{2} \ln \sigma^{2}-\frac{1}{2 \sigma^{2}} \sum_{n=1}^{N}\left(x_{n}-\mu\right)^{2}\right]$

$$
\hat{\mu}: \frac{\mathrm{d}}{\mu} \ln p(\mathcal{D} \mid \mu)=0
$$$$
\hat{\sigma}^{2}: \frac{\mathrm{d}}{\sigma^{2}} \ln p\left(\mathcal{D} \mid \sigma^{2}\right)=0
$$ 


\section{Inference for the Gaussian}

\section{Maximum likelihood}




\section{Inference for the Gaussian Maximum likelihood}

- Maximum likelihood solutions

$$
\begin{aligned}
\mu_{\mathrm{ML}} & =\frac{1}{N} \sum_{n=1}^{N} x_{n} \\
\sigma_{\mathrm{ML}}^{2} & =\frac{1}{N} \sum_{n=1}^{N}\left(x_{n}-\mu_{M L}\right)^{2}
\end{aligned}
$$

Equivalent to common mean and variance estimators (almost). 


\section{Inference for the Gaussian Maximum likelihood}

- Maximum likelihood solutions

$$
\begin{aligned}
\mu_{\mathrm{ML}} & =\frac{1}{N} \sum_{n=1}^{N} x_{n} \\
\sigma_{\mathrm{ML}}^{2} & =\frac{1}{N} \sum_{n=1}^{N}\left(x_{n}-\mu_{M L}\right)^{2}
\end{aligned}
$$

Equivalent to common mean and variance estimators (almost).

- Maximum likelihood ignores parameter uncertainty

- Think of the ML solution for a single observed datapoint $x_{1}$

$$
\begin{aligned}
\mu_{\mathrm{ML} 1} & =x_{1} \\
\sigma_{\mathrm{ML} 1}^{2} & =\left(x_{1}-\mu_{M L 1}\right)^{2}=0
\end{aligned}
$$




\section{Inference for the Gaussian Maximum likelihood}

- Maximum likelihood solutions

$$
\begin{aligned}
& \mu_{\mathrm{ML}}=\frac{1}{N} \sum_{n=1}^{N} x_{n} \\
& \sigma_{\mathrm{ML}}^{2}=\frac{1}{N} \sum_{n=1}^{N}\left(x_{n}-\mu_{M L}\right)^{2}
\end{aligned}
$$

Equivalent to common mean and variance estimators (almost).

- Maximum likelihood ignores parameter uncertainty

- Think of the ML solution for a single observed datapoint $x_{1}$

$$
\begin{aligned}
\mu_{\mathrm{ML} 1} & =x_{1} \\
\sigma_{\mathrm{ML} 1}^{2} & =\left(x_{1}-\mu_{M L 1}\right)^{2}=0
\end{aligned}
$$

- How about Bayesian inference? 


\section{Bayesian Inference for the Gaussian} Ingredients

- Data

$$
\mathcal{D}=\left\{x_{1}, \ldots, x_{N}\right\}
$$

- Model HGguss - Gaussian PDF

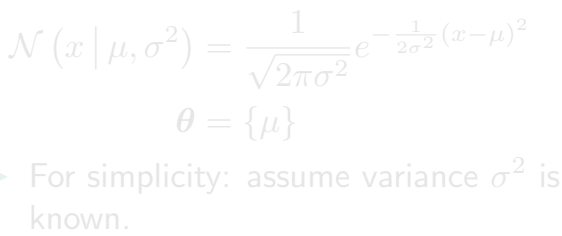

Likelihood 


\section{Bayesian Inference for the Gaussian}

\section{Ingredients}

- Data

$$
\mathcal{D}=\left\{x_{1}, \ldots, x_{N}\right\}
$$

- Model $\mathcal{H}_{\text {Gauss }}$ - Gaussian PDF

$$
\begin{aligned}
\mathcal{N}\left(x \mid \mu, \sigma^{2}\right) & =\frac{1}{\sqrt{2 \pi \sigma^{2}}} e^{-\frac{1}{2 \sigma^{2}}(x-\mu)^{2}} \\
\boldsymbol{\theta} & =\{\mu\}
\end{aligned}
$$

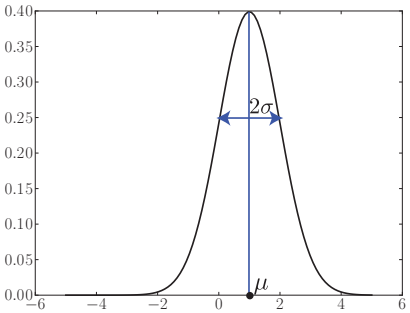

- For simplicity: assume variance $\sigma^{2}$ is known. 


\section{Bayesian Inference for the Gaussian}

\section{Ingredients}

- Data

$$
\mathcal{D}=\left\{x_{1}, \ldots, x_{N}\right\}
$$

- Model $\mathcal{H}_{\text {Gauss }}$ - Gaussian PDF

$$
\begin{aligned}
\mathcal{N}\left(x \mid \mu, \sigma^{2}\right) & =\frac{1}{\sqrt{2 \pi \sigma^{2}}} e^{-\frac{1}{2 \sigma^{2}}(x-\mu)^{2}} \\
\boldsymbol{\theta} & =\{\mu\}
\end{aligned}
$$

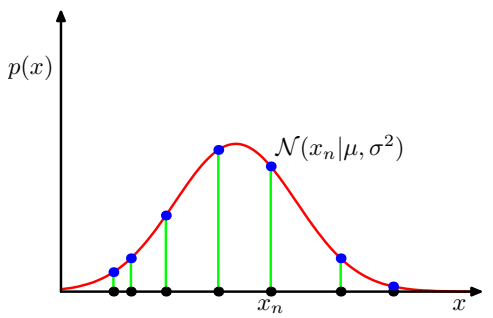

- Likelihood

$$
p(\mathcal{D} \mid \mu)=\prod_{n=1}^{N} \mathcal{N}\left(x_{n} \mid \mu, \sigma^{2}\right)
$$




\section{Bayesian Inference for the Gaussian}

\section{Bayes rule}

- Combine likelihood with a Gaussian prior over $\mu$

$$
p(\mu)=\mathcal{N}\left(\mu \mid m_{0}, s_{0}^{2}\right)
$$

- The posterior is proportional to

$$
p\left(\mu \mid \mathcal{D}, \sigma^{2}\right) \propto p\left(\mathcal{D} \mid \mu, \sigma^{2}\right) p(\mu)
$$




\section{Bayesian Inference for the Gaussian}

$$
\begin{aligned}
& p\left(\mu \mid \mathcal{D}, \sigma^{2}\right) \propto p(\mathcal{D} \mid \mu) p(\mu) \\
& =\left[\prod_{n=1}^{N} \frac{1}{\sqrt{2 \pi \sigma^{2}}} e^{-\frac{1}{2 \sigma^{2}}\left(x_{n}-\mu\right)^{2}}\right] \frac{1}{\sqrt{2 \pi s_{0}^{2}}} e^{-\frac{1}{2 s_{0}^{2}}\left(\mu-m_{0}\right)^{2}} \\
& =\underbrace{{\frac{1}{\sqrt{2 \pi \sigma^{2}}}}^{N} \frac{1}{\sqrt{2 \pi s_{0}^{2}}}}_{C 1} \exp \left[-\frac{1}{2 s_{0}^{2}}\left(\mu^{2}-2 \mu m_{0}+m_{0}^{2}\right)-\frac{1}{2 \sigma^{2}} \sum_{n=1}^{N}\left(\mu^{2}-2 \mu x_{n}+x_{n}^{2}\right)\right] \\
& =C 2 \exp [-\frac{1}{2} \underbrace{\left(\frac{1}{s_{0}^{2}}+\frac{N}{\sigma^{2}}\right)}_{1 / \hat{\sigma}}(\mu^{2}-2 \mu \underbrace{\hat{\sigma}\left(\frac{1}{s_{0}^{2}} m_{0}+\frac{1}{\sigma^{2}} \sum_{n=1}^{N} x_{n}\right)}_{\hat{\mu}})+C 3]
\end{aligned}
$$




\section{Bayesian Inference for the Gaussian}

$$
\begin{aligned}
& p\left(\mu \mid \mathcal{D}, \sigma^{2}\right) \propto p(\mathcal{D} \mid \mu) p(\mu) \\
& =\left[\prod_{n=1}^{N} \frac{1}{\sqrt{2 \pi \sigma^{2}}} e^{-\frac{1}{2 \sigma^{2}}\left(x_{n}-\mu\right)^{2}}\right] \frac{1}{\sqrt{2 \pi s_{0}^{2}}} e^{-\frac{1}{2 s_{0}^{2}}\left(\mu-m_{0}\right)^{2}} \\
& =\underbrace{\frac{1}{\sqrt{2 \pi \sigma^{2}}}{ }^{N} \frac{1}{\sqrt{2 \pi s_{0}^{2}}}}_{C 1} \exp \left[-\frac{1}{2 s_{0}^{2}}\left(\mu^{2}-2 \mu m_{0}+m_{0}^{2}\right)-\frac{1}{2 \sigma^{2}} \sum_{n=1}^{N}\left(\mu^{2}-2 \mu x_{n}+x_{n}^{2}\right)\right] \\
& =C 2 \exp [-\frac{1}{2} \underbrace{\left(\frac{1}{s_{0}^{2}}+\frac{N}{\sigma^{2}}\right)}_{1 / \hat{\sigma}}(\mu^{2}-2 \mu \underbrace{\hat{\sigma}\left(\frac{1}{s_{0}^{2}} m_{0}+\frac{1}{\sigma^{2}} \sum_{n=1}^{N} x_{n}\right)}_{\hat{\mu}})+C 3]
\end{aligned}
$$

- Posterior parameters follow as the new coefficients. 


\section{Bayesian Inference for the Gaussian}

$$
\begin{aligned}
& p\left(\mu \mid \mathcal{D}, \sigma^{2}\right) \propto p(\mathcal{D} \mid \mu) p(\mu) \\
& =\left[\prod_{n=1}^{N} \frac{1}{\sqrt{2 \pi \sigma^{2}}} e^{-\frac{1}{2 \sigma^{2}}\left(x_{n}-\mu\right)^{2}}\right] \frac{1}{\sqrt{2 \pi s_{0}^{2}}} e^{-\frac{1}{2 s_{0}^{2}}\left(\mu-m_{0}\right)^{2}} \\
& =\underbrace{\frac{1}{\sqrt{2 \pi \sigma^{2}}}{ }^{N} \frac{1}{\sqrt{2 \pi s_{0}^{2}}}}_{C 1} \exp \left[-\frac{1}{2 s_{0}^{2}}\left(\mu^{2}-2 \mu m_{0}+m_{0}^{2}\right)-\frac{1}{2 \sigma^{2}} \sum_{n=1}^{N}\left(\mu^{2}-2 \mu x_{n}+x_{n}^{2}\right)\right] \\
& =C 2 \exp [-\frac{1}{2} \underbrace{\left(\frac{1}{s_{0}^{2}}+\frac{N}{\sigma^{2}}\right)}_{1 / \hat{\sigma}}(\mu^{2}-2 \mu \underbrace{\hat{\sigma}\left(\frac{1}{s_{0}^{2}} m_{0}+\frac{1}{\sigma^{2}} \sum_{n=1}^{N} x_{n}\right)}_{\hat{\mu}})+C 3]
\end{aligned}
$$

- Posterior parameters follow as the new coefficients.

- Note: All the constants we dropped on the way yield the model evidence: $p\left(\mu \mid \mathcal{D}, \sigma^{2}\right)=\frac{p(\mathcal{D} \mid \mu) p(\mu)}{Z}$ 


\section{Bayesian Inference for the Gaussian}

- Posterior of the mean: $p\left(\mu \mid \mathcal{D}, \sigma^{2}\right) \propto \mathcal{N}(\mu \mid \hat{\mu}, \hat{\sigma})$, after some rewriting

$$
\begin{aligned}
\hat{\mu} & =\frac{\sigma^{2}}{N s_{0}^{2}+\sigma^{2}} m_{0}+\frac{N s_{0}^{2}}{N s_{0}^{2}+\sigma^{2}} \mu_{\mathrm{ML}}, \quad \mu_{\mathrm{ML}}=\frac{1}{N} \sum_{n=1}^{N} x_{n} \\
\frac{1}{\hat{\sigma}^{2}} & =\frac{1}{s_{0}^{2}}+\frac{N}{\sigma^{2}}
\end{aligned}
$$

- Limiting cases for no and infinite amount of data

\begin{tabular}{c|cc} 
& $N=0$ & $N \rightarrow \infty$ \\
\hline$\hat{\mu}$ & $m_{0}$ & $\mu_{\mathrm{ML}}$ \\
$\hat{\sigma}^{2}$ & $s_{0}^{2}$ & 0
\end{tabular}




\section{Bayesian Inference for the Gaussian}

\section{Examples}

- Posterior $p\left(\mu \mid \mathcal{D}, \sigma^{2}\right)$ for increasing data sizes.

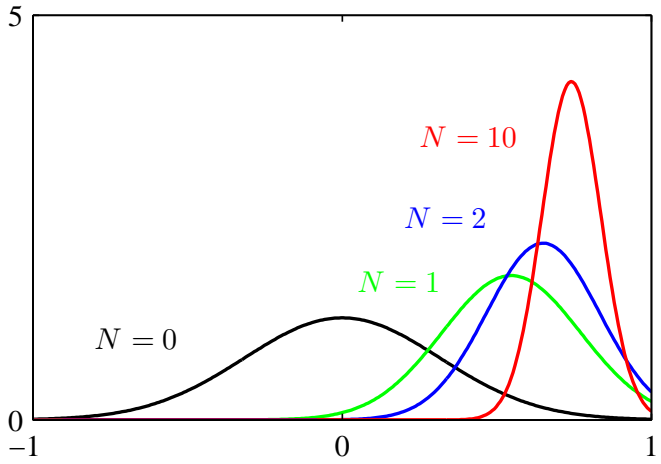

(C.M. Bishop, Pattern Recognition and Machine Learning) 


\section{Conjugate priors}

- It is not chance that the posterior

$$
p\left(\mu \mid \mathcal{D}, \sigma^{2}\right) \propto p\left(\mathcal{D} \mid \mu, \sigma^{2}\right) p(\mu)
$$

is tractable in closed form for the Gaussian. 


\section{Conjugate priors}

- It is not chance that the posterior

$$
p\left(\mu \mid \mathcal{D}, \sigma^{2}\right) \propto p\left(\mathcal{D} \mid \mu, \sigma^{2}\right) p(\mu)
$$

is tractable in closed form for the Gaussian.

\section{Conjugate prior}

$p(\theta)$ is a conjugate prior for a particular likelihood $p(\mathcal{D} \mid \theta)$ if the posterior is of the same functional form than the prior. 


\section{Conjugate priors}

\section{Exponential family distributions}

- A large class of probability distributions are part of the exponential family (all in this course) and can be written as:

$$
p(\mathbf{x} \mid \boldsymbol{\theta})=h(\mathbf{x}) g(\boldsymbol{\theta}) \exp \left\{\boldsymbol{\theta}^{\mathrm{T}} \mathbf{u}(\mathbf{x})\right\}
$$

- For example for the Gaussian:

$$
\begin{aligned}
& p\left(x \mid \mu, \sigma^{2}\right)=\frac{1}{2 \pi \sigma^{2}} \exp \left\{-\frac{1}{2 \sigma^{2}}\left(x^{2}-2 x \mu+\mu^{2}\right)\right\} \\
& =h(x) g(\boldsymbol{\theta}) \exp \left\{\boldsymbol{\theta}^{\mathrm{T}} \mathbf{u}(\mathbf{x})\right\} \\
& \text { with } \boldsymbol{\theta}=\left(\begin{array}{c}
\mu / \sigma^{2} \\
-1 / 2 \sigma^{2}
\end{array}\right), h(x)=\frac{1}{\sqrt{2 \pi}} \\
& \mathbf{u}(x)=\left(\begin{array}{c}
x \\
x^{2}
\end{array}\right), g(\boldsymbol{\theta})=\left(-2 \theta_{2}\right)^{1 / 2} \exp \left(\frac{\theta_{1}^{2}}{4 \theta_{2}}\right)
\end{aligned}
$$




\section{Conjugate priors \\ Exponential family distributions}

Conjugacy and exponential family distributions

- For all members of the exponential family it is possible to construct a conjugate prior.

- Intuition: The exponential form ensures that we can construct a prior that keeps its functional form. 


\section{Conjugate priors \\ Exponential family distributions}

\section{Conjugacy and exponential family distributions}

- For all members of the exponential family it is possible to construct a conjugate prior.

- Intuition: The exponential form ensures that we can construct a prior that keeps its functional form.

- Conjugate priors for the Gaussian $\mathcal{N}\left(x \mid \mu, \sigma^{2}\right)$

- $p(\mu)=\mathcal{N}\left(\mu \mid m_{0}, s_{0}^{2}\right)$

- $p\left(\frac{1}{\sigma^{2}}\right)=\Gamma\left(\frac{1}{\sigma^{2}}, a_{0}, b_{0}\right)$. 


\section{Bayesian Inference for the Gaussian Sequential learning}

- Bayes rule naturally leads itself to sequential learning

- Assume one by one multiple datasets become available: $\mathcal{D}_{1}, \ldots, \mathcal{D}_{S}$

$$
\begin{aligned}
& p_{1}(\boldsymbol{\theta}) \propto p\left(\mathcal{D}_{1} \mid \boldsymbol{\theta}\right) p(\boldsymbol{\theta}) \\
& p_{2}(\boldsymbol{\theta}) \propto p\left(\mathcal{D}_{2} \mid \boldsymbol{\theta}\right) p_{1}(\boldsymbol{\theta})
\end{aligned}
$$

- Note: Assuming the datasets are independent, sequential updates and a single learning step yield the same answer. 


\section{Outline}

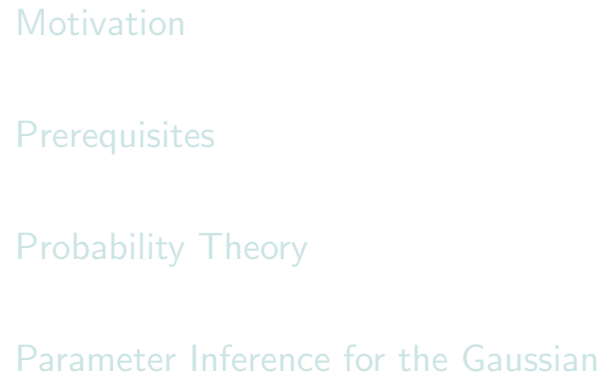

Summary 
- Probability theory: the language of uncertainty.

- Key rules of probability: sum rule, product rule.

- Bayes rules formes the fundamentals of learning. (posterior $\propto$ likelihood · prior).

- The entropy quantifies uncertainty.

- Parameter learning using maximum likelihood.

- Bayesian inference for the Gaussian. 